\title{
Supporting Undergraduates
} in Conducting Field-Based Research: A Perspective from On-Site Faculty and Staff

Field-based research programs offer students a singular opportunity to understand that today there are no simple scientific, economic or socio-political answers to the complex questions facing governments, communities, and local organizations. Through their research, students can gain a first-hand appreciation that decision making in the real world is a mix of all these disciplines, and that they have a vital role to play in participating in this process.

According to the most recent Open Doors report (2006), issued by the Institute of International Education, about 206,000 US students studied abroad in 2004/5. While about 55\% studied in Europe, an increasing number studied in other host countries around the world. Social science and physical science students comprised about $30 \%$ of all US study abroad students in this period. While study abroad programs encompassing a field research component are still in the minority, an increasing number of home institutions and field-based providers are supporting and conducting these types of programs.

As the student papers in this Special Issue of Frontiers demonstrate, there is high quality work being produced by undergraduates in settings as diverse as France, Thailand, Kenya, South Africa and Mali.

For these students this opportunity was likely a new experience, involving living and studying in international settings; dealing with language and culture differences; matriculating in programs operated by host country universities, independent program providers, or their home institution's international program; and learning how to conduct research that meets professional standards.

Much has been written and discussed regarding pre-departure orientation of US students studying abroad, along with studies and evaluations of the study abroad experience. Less discussion and research has focused on the experiences of the on-site faculty and staff who host students and incorporate field-based research into their courses and programs. These courses and programs involving student research include the following types:

- International university-based research, in which the student conducts research on a topic as part of a course or term paper; 
- Independent field-based research, in which the student identifies a topic, organizes the project, and conducts the field work, analysis, write-up, etc. for an overall grade;

- Collective field-based research, in which students, working under the guidance of a professor (either US or international), conduct a research project as part of a US-based course, or complementary to the professor's research focus;

- Client-focused, directed, field-based research in which the research conducted is in response to, or in collaboration with, a specific client ranging from an NGO, to a corporation, to an indigenous community, or a governmental agency.

The purpose of this article is to describe some of the issues and challenges that on-site faculty and staff encounter in preparing and supporting US undergraduate students to conduct formal research projects in international settings in order to maximize their success and the quality of their research. The perspectives described below have been gathered through informal surveys with a range of international program faculty and staff; discussions with program managers and faculty; and through our own experience at The School for Field Studies (SFS), with its formal directed research model. The survey sought responses in the following areas, among others: preparing students to conduct successfully their field-based research in a different socio-cultural environment; the skill building needs of students; patterns of personal, cultural, and/or technical challenges that must be addressed to complete the process successfully; and, misconceptions that students have about field-based research.

\section{Student Preparation}

Students work either individually or in groups to conduct their research, depending on the program. In either case on-site faculty and staff focus immediately on training students on issues ranging from personal safety and risk management, to cultural understanding, language training, and appropriate behavior. In programs involving group work, faculty and staff have learned that good teamwork dynamics cannot be taken for granted. They work actively with students in helping them understand the ebb and flow of groups, the mutual respect which must be extended, and the active participation that each member must contribute. As one on-site director indicates, "Students make their experience what it is through their behavior. We talk a lot about respecting each other as individuals and working together to make the project a great experience." 
Cultural and sensitivity training are a major part of these field-based programs. It is critical that students learn and appreciate the social and cultural context in which they will conduct their research. As another on-site director states, "It is most important that the students understand the context in which the research is happening. They need to know the values and basic cultural aspects around the project they will be working on. It is not simply doing 'good science.' It requires understanding the context so the science research reaches its goal.”

On-site faculty and staff also stress the importance of not only understanding cultural dynamics, but also acting appropriately and sensitively relative to community norms and expectations. Language training is also a component of many of these programs. As a faculty member comments, "Students usually need help negotiating a different culture and a new language. We try to help the students understand that they need to identify appropriate solutions for the culture they are in, and that can be very difficult at times."

\section{S k i I I B u i I d i n g}

Training students on the technical aspects of conducting field-based research is the largest challenge facing most on-site faculty and staff, who are often struck by the following:

- A high percentage of students come to these programs with a lack of knowledge of statistics and methods. They've either had very little training in statistics, or they find that real world conditions complicate their data. According to one faculty member, "Statistics are a big struggle for most students. Some have done a class, but when they come to work with real data it is seldom as black and white as a text book example and that leads to interpretation issues and lack of confidence in their data. They learn that ecology (for example) is often not clear, but that is OK."

- Both physical and social science students need basic training in scientific methodology in order to undertake their projects. Even among science majors there is a significant lack of knowledge of how to design, manage and conduct a research project. As a program director states, "Many students begin by thinking that field research is comprised only of data collection. We intensively train students to understand that good research is a process that begins with conceptualization of issues, moves into review of relevant literature, structures a research hypothesis, determines indicators and measurements, creates the research design, collects data, undertakes analysis and inference. This is followed by 
write-up in standard scientific format for peer review and input. This leads to refining earlier hypotheses, raising new questions and initiating further research to address new questions." Consistently, on-site faculty have indicated that helping students understand and appreciate this cycle is a major teaching challenge, but one that is critical to their education and the success of their various field research projects.

- The uncertainty and ambiguity that are often present in field research creates challenges for many students who are used to seeking 'the answer in the book.' On-site faculty help students understand that science is a process in which field-based research is often non-linear and prone to interruption by natural and political events. It is a strong lesson for students when research subjects, be they animal or human, don't cooperate by failing to appear on time, or at all, and when they do appear they may have their own agendas. Finally, when working with human communities, student researchers need to understand that their research results and recommendations are not likely to result in immediate action. Program faculty help them to understand that the real world includes politics, conflicting attitudes, regulatory issues, funding issues, and other community priorities.

- Both physical and social science students demonstrate a consistent lack of skill in technical and evidence-based writing. For many this type of writing is completely new and is a definite learning experience. As a faculty member states, "Some students find the report writing process very challenging. We want them to do well, but we don't want to effectively write their paper for them."

\section{Ch a I l e nges}

The preceding points address some of the technical work that on-site faculty conduct with students. Faculty also witness and experience the 'emotional' side of field based research being conducted by their students. This includes what one faculty member calls "a research-oriented motivation" — the need for students to develop a strong, energized commitment to overcome all the challenges necessary to get the project done. As another professor indicates, "At the front end the students don't realize how much effort they will have to expend because they usually have no experience with this sort of work before they do their project."

Related to this is the need for students to learn that flexibility in the research process does not justify a sloppy or casual approach. It does mean a recognition that human, political, and meteorological factors may intervene, requiring 
the ability to adapt to changed conditions. The goal is to get the research done. The exact mechanics for doing so will emerge as the project goes on.

"Frustration tolerance" is critical in conducting this type of work. Students have the opportunity to learn that certain projects need to incorporate a substantial window of time while a lengthy ethics approval and permit review system is conducted by various governmental agencies. Students learn that bureaucracies move at their own pace, and for reasons that may not be obvious.

Finally, personal challenges to students may include being uncomfortable in the field (wet, hot, covered in scrub itch) or feeling over-tired. As a faculty member states, "Many have difficulty adjusting to the early mornings my projects usually involve."

These issues represent a range of challenges that field-based research faculty and staff encounter in working with undergraduate students in designing and conducting their research projects around the world. In my own experience with SFS field-based staff, and in discussions with a wide variety of others who work and teach on-site, I am consistently impressed by the dedication, energy and commitment of these men and women to train, support and mentor students to succeed. As an on-site director summarizes, essentially speaking for all, "Fortunately, most of the students attending our program are very enthusiastic learners, take their limitations positively, and hence put tremendous effort into acquiring the required skills to conduct quality research."

\section{S u m m a r y / Conclusions}

Those international program faculty and staff who have had years of experience in dealing with and teaching US undergraduates are surprised that the US educational system has not better prepared students on subjects including statistics, scientific report formatting and composition, and research methodologies. They find that they need to address these topics on an intensive basis in order for a substantial number of students to then conduct their research work successfully. Having said this, on-site faculty and staff are generally impressed by the energy and commitment that most students put into learning the technical requirements of a research project and carrying it out to the best of their abilities.

Having students conduct real field-based research, and grading these efforts, is a very concrete method of determining the seriousness with which a student has participated in their study abroad program. Encouraging fieldbased research is good for students and good for study abroad because it has the potential of producing measurable products based on very tangible efforts. 
In a number of instances students have utilized their field research as the basis for developing their senior thesis or honors project back on their home campus. Successful field research has also formed the basis of Fulbright or Watson proposals, in addition to other fellowships and graduate study projects. An increasing number of students are also utilizing their field research, often in collaboration with their on-site program faculty, to create professional conference presentations and posters.

Some of these field-based research models also produce benefits for incountry clients, including NGOs, corporations and community stakeholders. In addition to providing the data, analyses, technical information, and recommendations that these groups might not otherwise be able to afford, it is a concrete mechanism for the student and her/his study abroad program faculty and staff to 'give back' to local stakeholders and clients. It changes the dynamic from the student solely asking questions, interviewing respondents, observing communities, to more of a mutually beneficial relationship. This is very important to students who are sensitive to this dynamic. It is also important to their program faculty and staff, and in most cases, genuinely appreciated by the local stakeholders. In essence, community identified and responsive research is an excellent mechanism for giving to a community — not just taking from it.

An increasing interest in conducting field-based research on the part of US universities and their students may have the effect of expanding the international destinations to which US students travel. A student's sociological, anthropological, or environmental interest and their desire to conduct field research in that academic discipline, for example, may help stretch the parameters of the student's comfort level to study in more exotic (non-traditional) locales.

Skill building in preparing for and conducting field-based research is an invaluable experience for the student's future academic and professional career. It is a fairly common experience for these students to indicate that with all the classroom learning they have done, their study abroad experience wherein they got their hands dirty, their comfort level stretched, their assumptions tested, and their work ethic challenged, provided them with an invaluable and life changing experience.

Conducting field-based research in an international setting provides real world experience, as the student papers in this edition of Frontiers attest. It also brings what may have only been academic subjects, like statistics, and research design and methodology, to life in a real-conditions context.

On a related note, conducting real field-based work includes the requirement to endure field conditions, remote locations, bad weather, personal 
discomforts, technological and mechanical breakdowns, and sometimes dangerous situations. Field research is hard work if it is done rigorously. In addition, field work often includes non-cooperating subjects that defy prediction, and may confound a neat research hypothesis. For a student considering a profession which requires a serious commitment to social or physical science field work this study abroad experience is invaluable. It clarifies for the student what is really involved, and it is helpful to the student in assessing their future career focus, as they ask the critical question — would I really want to do this as a fulltime career?

US education needs to bridge better the gap between the physical and social sciences. Students are done a disservice with the silo-type education that has been so prevalent in US education. In the real world there are no strictly scientific, economic, or sociological solutions to complex, vexing problems facing the global community. Going forward there needs to be interdisciplinary approaches to these issues by decision makers at all levels. We need to train our students to comprehend that while they may not be an ecologist, or an economist, or a sociologist, they need to understand and appreciate that all these perspectives are important and must be considered in effective decision making processes.

In conclusion, education abroad programs involving serious field-based research are not a distraction or diversion from the prescribed course of study at US home institutions; rather, they are, if done well, capable of providing real, tangible skills and experience that students lack, in spite of their years of schooling. This is the reward that is most meaningful to the international program faculty and staff who teach, mentor and support US students in conducting their field-based research activities. As an Australian on-site program director stated, "there are relatively few students who are adequately skilled in these (field research) areas when they come to our program. Most need a lot of instruction and assistance to complete their research projects, but that of course is part of what we're all about - helping students acquire or improve these critical skills." This is the real service that these programs and on-site faculty and staff offer to US undergraduates.

Paul Houlihan, President The School for Field Studies 
